\title{
Zigzag Faceting and Width Refinement of Graphene Nanoribbons and Nanoperforations via Catalyzed Edge-Annealing on $\mathrm{Cu}(111)$
}

Nathaniel S. Safron, Jonathan W. Choi, Myungwoong Kim, Naechul Shin, Padma Gopalan, and Michael S. Arnold*(*contact at msarnold@wisc.edu)

Department of Materials Science and Engineering, University of Wisconsin-Madison, Madison, WI 53706.

\begin{abstract}
:
Top-down subtractive lithography has previously been used to pattern graphene nanostructures which lack ideal properties due to (1) limited resolution and (2) disordered edges. Here, we introduce a method to convert such disordered edges into relatively smooth zigzag edges via annealing on a $\mathrm{Cu}(111)$ substrate at $\sim 950{ }^{\circ} \mathrm{C}$. The $\mathrm{Cu}$ catalyzes the re-arrangement of graphene edge atoms to energetically favorable sites, inducing zigzag edge faceting. The dimensions of the graphene nanostructures can be increased, decreased, or held constant during the annealing by tuning the relative balance between growth and etching reactions, described by a fundamental growth rate equation. To demonstrate the flexibility of this method, we lithographically pattern graphene nanoribbons with zigzag or armchair orientations, or alternatively perforate graphene with circular holes, and then anneal these nanostructures to realize zigzag edge termination in each case, with nanostructure feature size tailored from 8 to $80 \mathrm{~nm}$. The annealed nanostructures have smoother zigzag edges ( $40 \%$ reduction in $1 \sigma$ line edge roughness), and Raman spectroscopy confirms that they have lower edge disorder than top-down patterned samples.
\end{abstract}


Graphene has received widespread attention due to its excellent mechanical, thermal, electrical, optical, and chemical properties. ${ }^{1,2}$ For many applications, a suitable method for patterning and defining edges in graphene with minimal line edge roughness (LER) and along specified crystallographic directions is necessary. ${ }^{3,4}$ For example, laterally confined armchair graphene nanoribbons (GNRs) can exhibit a band gap enabling high conductance modulation at room temperature for transistor logic applications. ${ }^{5}$ Alternatively, smooth zigzag orientation GNRs have a high mid-gap density of states at their edges suitable for metallic interconnects, ${ }^{6,7}$ antiferromagnetic spin orientation at opposing edges with applications in spintronics, ${ }^{8}$ and high reactivity ${ }^{9}$ for chemical functionalization. ${ }^{10}$

Thus far, the highest fidelity graphene nanostructures have been produced by bottom-up methods. ${ }^{11-}$ ${ }^{16}$ However, these methods have not allowed for arbitrary control over graphene nanostructure size, shape, placement, density, and organization, all of which are necessary for creating complex circuits. Top-down subtractive lithography allows for this arbitrary control. Large flakes or continuous sheets of monolayer graphene can be patterned into arbitrary geometries via optical, electron beam, ${ }^{3}$ block copolymer, ${ }^{17,} 18$ nanoimprint, ${ }^{19}$ and other self-assembly lithographies. ${ }^{20}$ The unwanted regions of graphene are etched away, for example via directional reactive ion etching. ${ }^{3,21}$ The challenge with top-down lithography is that this etching yields edges that are not atomically smooth and that can be undesirably chemically functionalized by the etchant. ${ }^{18,22}$ For example, graphene that is patterned using block copolymers has demonstrated a LER of $\sim 1.2 \mathrm{~nm}$ (measured over a $170 \mathrm{~nm}$ length-scale). ${ }^{23}$ Edge disorder is problematic because it drastically lowers charge transport mobility, ${ }^{21,24,25}$ perturbs edge transport phenomena, and gives rise to localized edge states that can energetically lie in the band gap of semiconducting forms of graphene nanostructures. $^{26-29}$

In this paper, we present a novel method to anisotropically remove etch-induced disorder from the edges of top-down patterned graphene nanostructures in order overcome this problem. Previous studies have shown that the edges of freestanding graphene and graphite will anneal and reorder with zigzag or armchair faceting at an extreme temperature of $\sim 2000{ }^{\circ} \mathrm{C} .{ }^{30}$ Our annealing is performed on a $\mathrm{Cu}$ thin film to catalyze edge reorganization at a drastically lower temperature of $\sim 950{ }^{\circ} \mathrm{C}$. We exploit processes that are 
inherent to graphene CVD on $\mathrm{Cu}$ substrates ${ }^{31}$ to drive the annealing, in particular, the fact that both growing and etching graphene domains show preference to the zigzag edge due to favorable energetics or kinetics. ${ }^{32-}$ 35

Moreover, whereas previous work has used etch-pit and over-etching phenomena ${ }^{36-38}$ to facet the edges of graphene nanostructures, here we use a new mechanism that does not necessitate dimensional change through which it is possible to increase, decrease, or hold constant the dimensions of the nanostructures by controlling the balance between growth and etching reactions. In particular, we have identified a hydrogen concentration dependent critical methane concentration (CMC) in which the net growth rate of graphene is zero, ${ }^{39}$ which occurs when intermediate hydrocarbons, generated from the decomposition of adsorbed $\mathrm{CH}_{4}$ on the $\mathrm{Cu}$, attach to graphene edges at the same rate as the graphene etches. By adjusting the $\mathrm{CH}_{4}$ concentration $\left(\left[\mathrm{CH}_{4}\right]\right)$ to above or below the $\mathrm{CMC}$, the graphene either grows or etches, respectively, at a precisely controlled rate.

The edge-annealing process is depicted in Figure 1. Beginning with a continuous epitaxially oriented graphene monolayer grown on $\mathrm{Cu}(111)$ on sapphire, we define GNRs of width, $w=75 \pm 8 \mathrm{~nm}$ (Fig. 1a), with their long-axis parallel to the zigzag direction, using e-beam lithography in conjunction with reactive ion etching (see Methods, Supplemental Information). The use of $\mathrm{Cu}(111)$ substrates instead of polycrystalline $\mathrm{Cu}$ foils is necessary so that all of the nanoribbons have the same crystallographic orientation and so that this crystallographic orientation is controlled. These top-down patterned nanoribbons have a $1 \sigma$ line edge roughness of $2.0 \mathrm{~nm}$ in the SEM (analyzed over a characteristic length-scale of 250 $\mathrm{nm}$ ), are functionalized with oxygen groups near the edges ${ }^{18,22}$ and likely contain some interior defects close to the edges as a byproduct of the reactive ion etching, ${ }^{21,40}$ as represented in Fig. 1a.

After top-down patterning, the GNRs are annealed on the $\mathrm{Cu}(111)$ films. By controlling the $\left[\mathrm{CH}_{4}\right]$ to $b e<,=$, or $>$ the $\mathrm{CMC}$, we tune the CVD condition to be etching + annealing, annealing only, or growing + annealing, respectively, and therefore control the final width of the GNRs. For example, in Fig. 1b, c and d, we have implemented the same anneal temperature, $T$ of $950{ }^{\circ} \mathrm{C}$, time $t$ of $25 \mathrm{~min}$, and $\left[\mathrm{H}_{2}\right]$ of $4.75 \%$, while varying only $\left[\mathrm{CH}_{4}\right]$ from 20 to 88 to $97 \mathrm{ppm}$, which results in a final $w$ of $25 \pm 4,75 \pm 9$, and $87 \pm 9$ 
$\mathrm{nm}$, respectively. In each case, the resulting GNRs have straight zigzag edges similar to the edges observed during the anisotropic growth or etching of large graphene crystals seen in literature ${ }^{32,34}$.

Regardless of the patterned edge orientation of the top-down graphene nanostructures, the edges always re-facet along zigzag orientations during annealing. Edges that already have zigzag orientation (Fig. 1a and Fig. 2a) remain zigzag oriented after annealing irrespective of if there is net etching (Fig. $1 \mathrm{~b}$ and Fig. 2d), if etching and growth are balanced (Fig. 1c), or if there is net growth (Fig. 1d). In contrast, edges with initially armchair orientation (Fig. 2b) reorient by $\pm 30^{\circ}$ during annealing, in many cases becoming jagged (Fig. 2e). In this latter case, the GNRs grow or are etched by about $25 \mathrm{~nm}$ in alternating regions so that the edges have zigzag orientation in roughly $50 \mathrm{~nm}$ lengths. The large amount of mass transfer in this case clearly demonstrates a preference for both growth and etching towards the zigzag edge.

To confirm the edge orientation, we perform TEM analysis. We begin with top-down patterned nanoperforated graphene (NPG), which has a periodic array of circular holes following top-down pattern transfer from a block copolymer template (Fig. 2c), where the smallest width between two holes is $w=15$ $\pm 3 \mathrm{~nm}$ (see Methods, Supplemental Information). After edge annealing, TEM imaging clearly shows that the circular holes in the NPG rearrange to form faceted hexagonal holes with $w=11 \pm 1 \mathrm{~nm}$ (Fig. 2f). The edge orientation is found through selected-area electron diffraction (SAED) and confirmed to be zigzag (see Fig. $2 \mathrm{f}$ insert for the SAED and Fig. S1 for the TEM image of the selected area from the diffraction pattern). The TEM analysis clearly demonstrates that arbitrary graphene nanostructures can be annealed to convert mixed edge type into zigzag.

The LER of GNRs before and after annealing for 25 minutes at the CMC is analyzed via SEM in Fig. S2 and measured over a range of length-scales varying from 100 to $300 \mathrm{~nm}$. The annealing process reduces the LER by about $40 \%$. For example, the LER is decreased from 2.0 to $1.2 \mathrm{~nm}$ at a measurement length of $250 \mathrm{~nm}$ while it is reduced from 1.4 to $0.9 \mathrm{~nm}$ at a measurement length of $100 \mathrm{~nm}$. Below a measurement length of $100 \mathrm{~nm}$, the LER decreases rapidly. For example, the LER of the faceted hexagonal holes in annealed NPG is only $0.16 \mathrm{~nm}$ over a measurement length of $10 \mathrm{~nm}$ (analyzed via TEM). 
Disorder in the graphene nanostructures is additionally quantified through Raman spectroscopy. The D-band $\left(1350 \mathrm{~cm}^{-1}\right)$ intensity, originating from electron scattering from internal defects and edges of the graphene crystal, is compared to the G-band $\left(1585 \mathrm{~cm}^{-1}\right)$ intensity, arising from the graphene bulk. The ratio of the scattering intensity, $I(D) / I(G)$, characterizes the concentration of defects and is found to scale with $1 / w$ as the edges comprise a larger fraction of the structures at smaller $w^{14}$ The full-width halfmaximum (FWHM) of the peaks is also quantified and is related to disorder in the material. ${ }^{41}$

The Raman spectra from top-down patterned $(w=56 \pm 2 \mathrm{~nm})$ and edge-annealed $(55 \pm 2 \mathrm{~nm}) \mathrm{GNR}$ arrays, respectively, are compared in Fig. 3a, where the former structures show a much larger $I(D) / I(G)$ ratio ( 0.68 compared to 0.27$)$ indicating a larger concentration of electron scattering defects. Additionally, the top-down patterned samples have a larger FWHM of the D- and G- peaks (33 and $45 \mathrm{~cm}^{-1}$ compared to 26 and $15 \mathrm{~cm}^{-1}$, respectively), indicating more disorder. We have compiled the $I(D) / I(G)$ ratio and FWHM of top-down patterned and edge-annealed $\left(T=950^{\circ} \mathrm{C}, t=25 \mathrm{~min}\right.$, varying $\left.\left[\mathrm{CH}_{4}\right]\right)$ graphene nanostructures as a function of $w$ in Fig. 3b-c and find that the edge-annealed samples clearly demonstrate a lower concentration of defects and less disorder at all $w$. The reduction in $I(D) / I(G)$ with annealing is about $40 \%$ at any given $w$ whereas the reduction in the number of edge atoms due to the decreased LER is only about $3 \%$, indicating that the annealing process must substantially reduce the concentration of internal defects ${ }^{21}$, ${ }^{40}$ near the edges. We hypothesize that the reduction in the FWHM following annealing can be at least partially ascribed to the removal of oxygen functionalization that exists prior to annealing, which has been shown to affect Raman peak positions in graphene via doping. ${ }^{42}$

The net growth rate, $G R$, during annealing is quantified in Fig. S3 as a function of CVD conditions and is determined from the initial and final widths of the GNR arrays and annealing time. Varying $\left[\mathrm{CH}_{4}\right]$ at fixed $T$ of $950{ }^{\circ} \mathrm{C}$ and $\left[\mathrm{H}_{2}\right]$ of $4.76 \%$, we find that:

$$
G R\left(\left[\mathrm{CH}_{4}\right]\right)=A\left(\left[\mathrm{CH}_{4}\right]-\mathrm{CMC}\right) / C M C
$$

where $A=3.2 \pm 0.2 \mathrm{~nm} / \mathrm{min}$ and $\mathrm{CMC}=88 \mathrm{ppm}$, which matches the functional form of the fundamental growth rate equation developed in our previous work. ${ }^{39}$ Equation [1] is important because it can be used to determine the $\left[\mathrm{CH}_{4}\right]$ necessary to etch, anneal, or grow at a specified rate. 
Setting the GR to $<0$ (etching + annealing) enables the width of GNRs to be controllably decreased. It is unclear, however, if it is better to etch quickly or slowly. To answer this question, we etch different sets of GNRs from $w=75 \mathrm{~nm}$ to $w=50 \mathrm{~nm}$, at different rates, controlled by tuning the $\left[\mathrm{CH}_{4}\right]$ (Fig. S4a-d). At slow etch rates with $\left[\mathrm{CH}_{4}\right]$ close to the $\mathrm{CMC}$, the average $w$ follows Eq. 1 but there is substantial ribbon to ribbon variation in $w$, and $w$ varies along the length of individual ribbons, as well (Fig. S4c-d). In contrast, more rapid etching at lower $\left[\mathrm{CH}_{4}\right]$ (Fig. S4a-b and as in Fig. 1b) results in less width polydispersity and yields well-ordered edges. For example, when $w$ is decreased from 75 to $50 \mathrm{~nm}$ over a duration of only 10 min, the $I(D) / I(G)$ ratio, normalized by the GNR width, decreases by approximately a factor of 2 (Fig. S4e). In contrast, the $I(D) / I(G)$ ratio increases during prolonged, slower annealing+etching. When $w$ is decreased from 75 to $50 \mathrm{~nm}$ over a duration that exceeds $60 \mathrm{~min}$, the $I(D) / I(G)$ ratio, normalized by the GNR width, instead increases with respect to $I(D) / I(G)$ prior to annealing. Thus, fast etching+annealing for short durations is more desirable.

Temperature is another important parameter that affects the fidelity of the annealing. Varying temperature in the range of $900-1000{ }^{\circ} \mathrm{C}$ under etching + annealing conditions, we find the process is exothermic with Arrhenius temperature dependence, with the growth rate coefficient from Equation $1, A \propto$ $e^{-E_{A} / k_{B} T}$, where $k_{B}$ is the Boltzmann constant and the fit activation energy $E_{A}$ is $2.6 \pm 0.3 \mathrm{eV}$ (Fig. S5a), in good agreement with the growth rate activation energy for $\mathrm{CVD}$ on $\mathrm{Cu}(111)$ in the literature. ${ }^{43}$ At $1000{ }^{\circ} \mathrm{C}$, the GNRs break and rotate, while for $T \leq 950{ }^{\circ} \mathrm{C}$, this problem is avoided (Fig. S5b-e). It is possible that the unsuccessful edge-annealing at $T=1000{ }^{\circ} \mathrm{C}$ is due to pre-melting of the $\mathrm{Cu}$ surface, ${ }^{44}$ which makes the GNRs mobile and may also explain why longer annealing at $T=950{ }^{\circ} \mathrm{C}$ results in more disordered GNR arrays, as noted in the previous paragraph. The $I(D) / I(G)$ ratio, normalized by width, increases by $\sim 30 \%$ as $T$ is decreased from 950 to $900{ }^{\circ} \mathrm{C}$, indicating that the annealing process yields more anisotropic edges at higher temperatures as long as $T \leq 950^{\circ} \mathrm{C}$.

To test electrical properties, we fabricate field effect transistors by transferring the nanostructured graphene from the $\mathrm{Cu}(111)$ to a $\mathrm{SiO}_{2}(89 \mathrm{~nm}) / \mathrm{Si}(\mathrm{p}++)$ substrate, where the substrate serves as both the gate dielectric and gate electrode (Fig. 4a,b), and measure low-field transconductance (see Methods, 
Supplemental Information). The room temperature hole mobility for an annealed array of $w=25 \pm 6 \mathrm{~nm}$ GNRs of $290 \pm 50 \mathrm{~cm}^{2} /$ Vs compares favorably with the top-down patterned array of $w=28 \pm 3 \mathrm{~nm}$ GNRs of $200 \pm 40 \mathrm{~cm}^{2} / \mathrm{Vs}$. Compiling data from all of our transistors, we find that the mobility roughly scales with $w^{2}$ (Fig. 4c), where the edge-annealed samples have slightly higher mobility at any specific $w$. The ON/OFF conductance modulation of all edge-annealed devices is in the range of $7-11$ irrespective of $w$, consistent with the band structure of semi-metallic zigzag GNRs.

The primary factor that limits charge transport mobility is not yet clear because several distinct electron scattering mechanisms can each potentially give rise to a mobility that scales as $w^{2}$, including LER scattering and charged impurity scattering from surface adsorbates. Our continuous graphene is charged impurity scattering-limited, potentially from a combination of physisorbed $\mathrm{H}_{2} \mathrm{O}$ and $\mathrm{O}_{2}$ species in ambient, $\mathrm{SiO}_{2}$ trap states, and polymer residue from $\operatorname{transfer}^{45}$ (see Discussion in Supplemental Information). It is entirely plausible that charged impurity scattering could be the limitation in our GNRs, as well. Future experiments will be needed in order to better differentiate between these two possible mechanisms and to quantify the intrinsic LER limited mobility of nanostructured graphene, before and after annealing.

In conclusion, the presented work demonstrates that edge-annealing is a clear route to (1) tailor the width of graphene nanostructures, either by growth or etching, and to (2) convert the edges of rough, lithographically defined nanostructures into smooth zigzag edges. This robust method is able to convert armchair orientation edges to zigzag ones (rotated by $30^{\circ}$ ) and circular holes to hexagonal ones, with smooth zigzag edges that are verified via TEM. We have isolated ideal process conditions in which the placement of GNRs remains unchanged and the line edge roughness and edge disorder decrease compared to topdown patterned nanostructures as identified by microscopy and Raman spectroscopy. We expect these results, combined with next generation state-of-the-art lithography, will enable the wafer scale patterning of narrow graphene nanostructures with smooth zigzag edges, suitable for many applications. 


\section{FIGURES:}
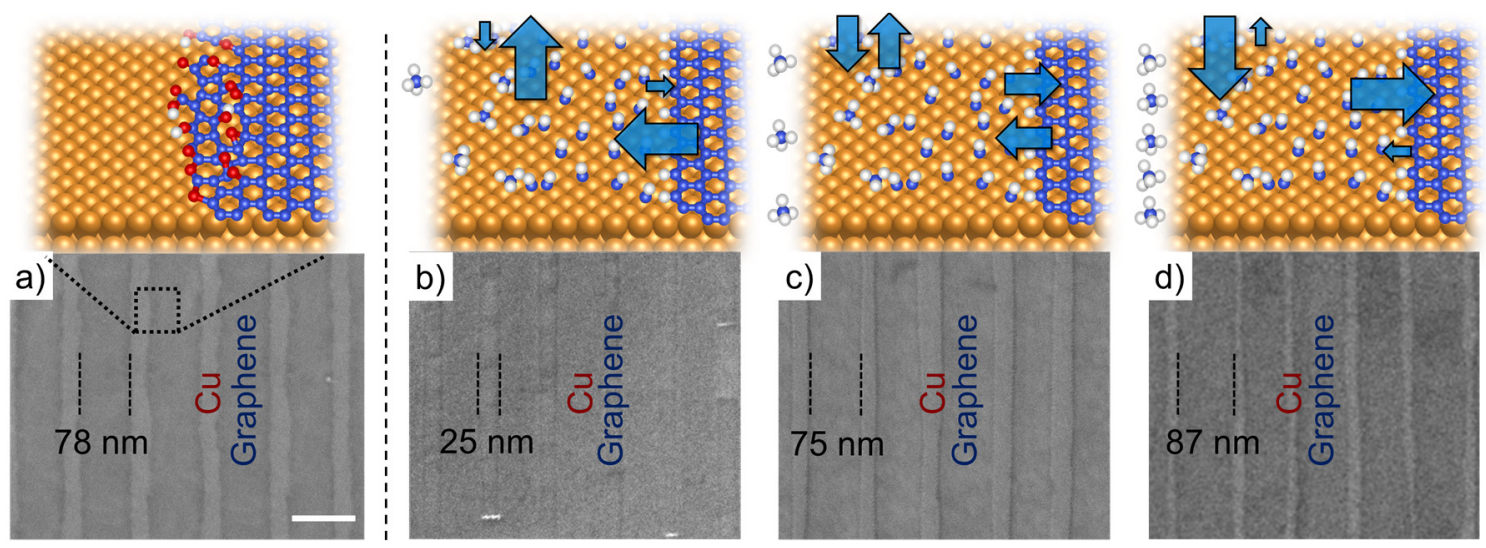

Figure 1. Schematic (top) and SEM (bottom) of GNRs a) after top-down patterning, and after edgeannealing under conditions of b) etching + annealing, c) annealing only and d) growth + annealing, conditions: $T=950^{\circ} \mathrm{C}, t=25 \mathrm{~min}$ for $\mathrm{b}-\mathrm{d},\left[\mathrm{CH}_{4}\right]=20,88$, and $97 \mathrm{ppm}$, for b, c, and d, respectively (SEM same magnification, scale bar $=100 \mathrm{~nm})$. 


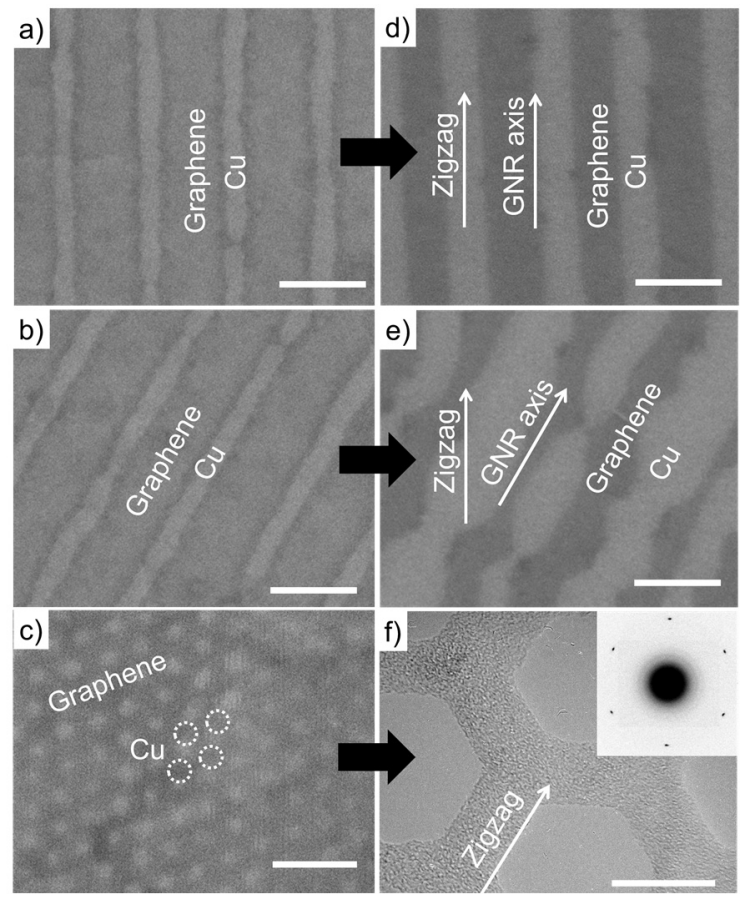

Figure 2. a-c) Top-down patterned graphene nanostructures. a) SEM of zigzag oriented GNRs, b) SEM of armchair oriented GNRs, and c) SEM of NPG. d-f) Edge annealed graphene nanostructures. d) SEM of zigzag-edged GNRs, e) SEM of rearranged armchair GNRs, and f) TEM of NPG, insert: SAED of region in Fig. S1, which includes region in $\mathrm{f}$. Scale bars $=100 \mathrm{~nm}$ in a-e and $20 \mathrm{~nm}$ in $\mathrm{f}$. 

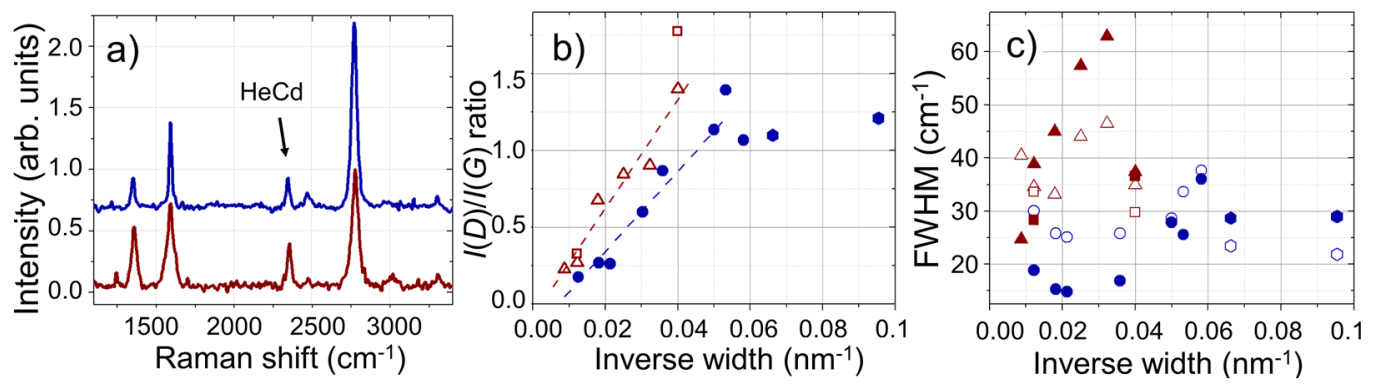

Figure 3. a) Raman spectra of edge-annealed $w=55 \pm 2 \mathrm{~nm}$ GNRs (top, blue) and top-down patterned $w=$ $56 \pm 2 \mathrm{~nm}$ GNRs (bottom, red), b) $I(D) / I(G)$ vs. 1/w for edge-annealed (blue, closed) zigzag GNRs (circles) and NPG (hexagons), compared to top-down (red, open) zigzag (triangles) and armchair (squares) oriented GNRs. Dashed lines are linear fits to zigzag GNRs, c) FWHM of the D-band (open) and G-band (closed) vs. $1 / w$ for edge-annealed (blue) and top-down (red) patterned graphene nanostructures, marker shapes same as b. 

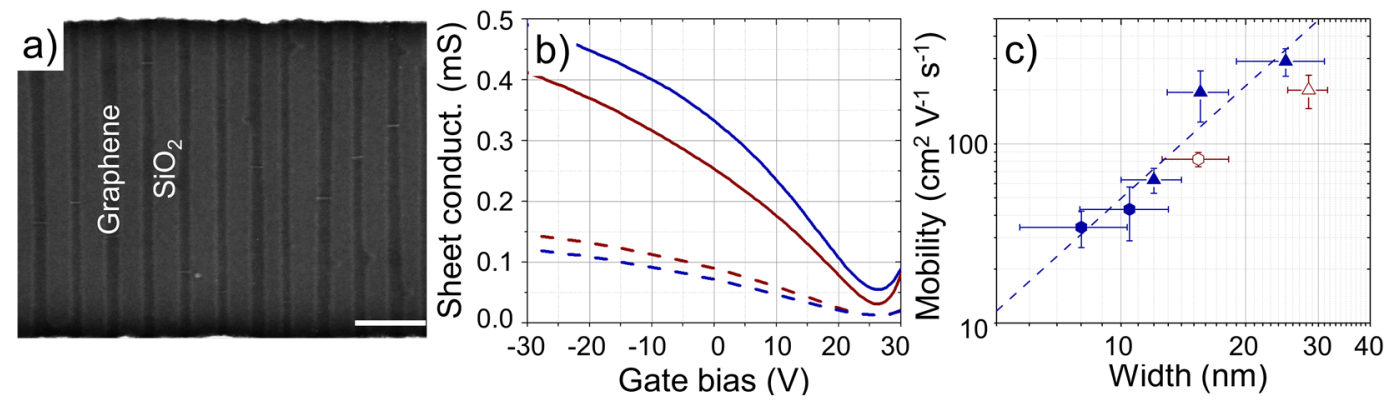

Figure 4. a) SEM image of $w=25 \pm 6 \mathrm{~nm}$ GNR device of sample from Fig. $1 \mathrm{~b}$ after transfer to $\mathrm{Si} / \mathrm{SiO}_{2}$ substrate (scale bar $=200 \mathrm{~nm}$ ). b) Sheet conductance vs. gate voltage for edge-annealed (blue) device in 4a (solid) and $w=10 \pm 3 \mathrm{~nm} \mathrm{NPG} \mathrm{(dashed)} \mathrm{device} \mathrm{compared} \mathrm{to} \mathrm{top-down} \mathrm{(red)} w=28 \pm 3 \mathrm{~nm}$ GNR device (solid) and $w=15 \pm 3 \mathrm{~nm} \mathrm{NPG} \mathrm{(dashed)} \mathrm{device.} \mathrm{c)} \mathrm{GNR} \mathrm{array} \mathrm{(triangles)} \mathrm{and} \mathrm{NPG} \mathrm{(circles)} \mathrm{hole} \mathrm{mobility}$ vs. $w$ for all edge-annealed (blue, closed) and top-down (red, open) samples, fit to $\mu \propto w^{2}$ shown as dashed line. 


\section{ACKNOWLDEGEMENTS:}

NSS, NS, and MSA acknowledge support from the DOE Office of Science Early Career Research Program (Grant number DE-SC0006414) through the Office of Basic Energy Sciences, for work on graphene synthesis, ebeam lithography, edge annealing, microscopy, Raman spectroscopy, and transport measurements. PG and JC acknowledge support from the National Science Foundation (Grant number CMMI-1129802) and MYK acknowledges support from the Wisconsin Alumni Research Foundation, for work on block copolymer patterning. 


\section{REFERENCES:}

1. Novoselov, K. S., et al. Nature 2005, 438, (7065), 197-200.

2. Wang, X. R., et al. Physical Review Letters 2008, 100, (20).

3. Han, M. Y., et al. Physical Review Letters 2007, 98, (20).

4. $\quad$ Yang, L., et al. Physical Review Letters 2007, 99.

5. Ezawa, M. Physical Review B 2006, 73, (4), 8.

6. Guo, J., et al. Nano Letters 2007, 7, (7), 1935-1940.

7. Li, Y. Y., et al. Nature Communications 2014, 5, 8.

8. $\quad$ Son, Y. W., et al. Nature 2006, 444, (7117), 347-349.

9. Baldoni, M., et al. Chemical Physics Letters 2008, 464, (4-6), 202-207.

10. Hod, O., et al. Nano Letters 2007, 7, (8), 2295-2299.

11. Li, X. L., et al. Science 2008, 319, (5867), 1229-1232.

12. Jiao, L. Y., et al. Nature 2009, 458, (7240), 877-880.

13. Cai, J. M., et al. Nature 2010, 466, (7305), 470-473.

14. Safron, N. S., et al. Advanced Materials 2012, 24, (8), 1041-1045.

15. Campos, L. C., et al. Nano Letters 2009, 9, (7), 2600-2604.

16. Sprinkle, M., et al. Nature Nanotechnology 2010, 5, (10), 727-731.

17. Bai, J. W., et al. Nature Nanotechnology 2010, 5, (3), 190-194.

18. Kim, M., et al. Nano Letters 2010, 10, (4), 1125-1131.

19. Liang, X. G., et al. Nano Letters 2010, 10, (7), 2454-2460.

20. Safron, N. S., et al. Small 2011, 7, (4), 492-498.

21. Kim, M., et al. Acs Nano 2012, 6, (11), 9846-9854.

22. Heydrich, S., et al. Appl. Phys. Lett. 2010, 97, (4).

23. Liang, X., et al. Acs Nano 2012, 6, (11), 9700-9710.

24. Mucciolo, E. R., et al. Physical Review B 2009, 79, (7).

25. Betti, A., et al. Ieee Transactions on Electron Devices 2011, 58, (9), 2824-2830.

26. Gallagher, P., et al. Physical Review B 2010, 81, (11).

27. Stampfer, C., et al. Physical Review Letters 2009, 102, (5).

28. Vanevic, M., et al. Physical Review B 2009, 80, (4), 8.

29. Kobayashi, Y., et al. Physical Review B 2006, 73, (12).

30. Jia, X. T., et al. Science 2009, 323, (5922), 1701-1705.

31. Li, X., et al. Science 2009, 324, (5932), 1312-1314.

32. Zhang, Y., et al. Acs Nano 2012, 6, (1), 126-132.

33. Yu, Q. K., et al. Nature Materials 2011, 10, (6), 443-449.

34. Luo, Z., et al. Acs Nano 2011, 5, (11), 9154-9160.

35. Shu, H., et al. Acs Nano 2012, 6, (4), 3243-3250.

36. Yang, R., et al. Advanced Materials 2010, 22, (36), 4014-4019.

37. Nemes-Incze, P., et al. Nano Res. 2010, 3, (2), 110-116.

38. Wang, X. R., et al. Nat. Chem. 2010, 2, (8), 661-665.

39. Safron, N. S., et al. Journal of Materials Chemistry C 2014, 2, (4), 744-755.

40. Ryu, S., et al. Acs Nano 2011, 5, (5), 4123-4130.

41. Cancado, L. G., et al. Nano Letters 2011, 11, (8), 3190-3196.

42. Das, A., et al. Nature Nanotechnology 2008, 3, (4), 210-215.

43. Kim, H., et al. Acs Nano 2012, 6, (4), 3614-3623.

44. Daff, T. D., et al. Surface Science 2009, 603, (3), 445-454.

45. Petrone, N., et al. Nano Letters 2012, 12, (6), 2751-2756. 